\title{
Computational design and experimental verification of a symmetric protein homodimer
}

\author{
Yun Mou ${ }^{a, 1}$, Po-Ssu Huang ${ }^{b, 1,2}$, Fang-Ciao Hsuc, Shing-Jong Huang ${ }^{\text {d,3 }}$, and Stephen L. Mayo ${ }^{a, e, 3}$ \\ a Division of Chemistry and Chemical Engineering, California Institute of Technology, Pasadena, CA 91125; ${ }^{\mathrm{b}}$ Biochemistry and Molecular Biophysics Option, \\ California Institute of Technology, Pasadena, CA 91125; 'Technology Commons, College of Life Science and Center for Systems Biology, National Taiwan

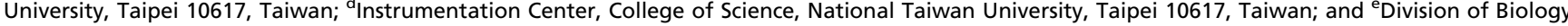 \\ and Biological Engineering, California Institute of Technology, Pasadena, CA 91125
}

Contributed by Stephen L. Mayo, May 18, 2015 (sent for review December 1, 2014)

\begin{abstract}
Homodimers are the most common type of protein assembly in nature and have distinct features compared with heterodimers and higher order oligomers. Understanding homodimer interactions at the atomic level is critical both for elucidating their biological mechanisms of action and for accurate modeling of complexes of unknown structure. Computation-based design of novel protein-protein interfaces can serve as a bottom-up method to further our understanding of protein interactions. Previous studies have demonstrated that the de novo design of homodimers can be achieved to atomic-level accuracy by $\beta$-strand assembly or through metal-mediated interactions. Here, we report the design and experimental characterization of a $\alpha$-helix-mediated homodimer with C2 symmetry based on a monomeric Drosophila engrailed homeodomain scaffold. A solution NMR structure shows that the homodimer exhibits parallel helical packing similar to the design model. Because the mutations leading to dimer formation resulted in poor thermostability of the system, design success was facilitated by the introduction of independent thermostabilizing mutations into the scaffold. This two-step design approach, function and stabilization, is likely to be generally applicable, especially if the desired scaffold is of low thermostability.
\end{abstract}

computational protein design | homodimer | docking |

nuclear magnetic resonance

D rotein-protein interactions play a central role in nearly all biological processes, including cell signaling, immune responses, regulation of transcription and translation, and cell-cell adhesion. Improving our understanding of protein-protein interactions is therefore an important component to advancements in both basic research and applications in the pharmaceutical, chemical, and biotechnology industries. The increasing availability of high-resolution structures has led to the identification of unique features of protein-protein interactions (1-3). Specific interfacial residues that contribute to most of the binding energy ("hot spots"), networks of hydrogen bonds, and shape complementarity have all been identified as important. These features have therefore been incorporated into many protein docking and protein design algorithms $(4,5)$. Protein docking algorithms have been used successfully to screen millions of docking positions and to identify the correct (near-native) structures (6). Computational design tools have also exploited our knowledge of protein-protein interactions to design enhanced affinity or altered specificity successfully $(7,8)$, to graft binding motifs onto a desired scaffold (9-11), and to create novel interfaces (12-20). Several studies have shown that it is possible to achieve atomiclevel accuracy in the de novo design of protein dimers $(12,13$, $16)$, as well as highly symmetric nanomaterials (17-20). However, the success rate of protein interface design is rather low (21), and protein interface modeling and design remain significant challenges (22).

Homodimers are the most common type of protein assembly and are well represented in the Protein Data Bank (PDB). Compared with heterodimers, homodimers have a larger surface area; fewer hydrogen bonds; higher hydrophobicity; and, typically, C2 symmetry (23). Although homodimers are abundant in nature, there are only a few examples of the computational design of symmetric homodimers. Stranges et al. (16) showed that solvent-exposed $\beta$-strands can be used as anchors to design a symmetric homodimer that associates via $\beta$-strand pairing. Der et al. (13) incorporated metal binding sites to drive homodimerization and achieve high affinity and orientation specificity. Interestingly, in their study, the helices on each side of the metalmediated homodimer interface aligned nearly orthogonally, unlike the parallel or antiparallel alignments of helices typically found in nature. Both parallel and antiparallel coiled-coil-like dimers have been designed using short peptides $(24,25)$. However, to our knowledge, there have been no structurally verified homodimers designed with an $\alpha$-helical interface. Helical interactions, often in the form of coiled-coils, occur twice as frequently at homodimer interfaces $(22.4 \%)$ compared with heterodimer interfaces $(10.9 \%)$, but $\beta$-strand- $\beta$-strand interactions are seen at about the same frequency ( $8.8 \%$ and $8.4 \%$, respectively) (26). These observations motivated our interest in the computational design of an $\alpha$-helix-mediated $\mathrm{C} 2$ symmetric homodimer based on a monomeric globular protein scaffold.

Designing a homodimer using $\alpha$-helical interactions presents many challenges. First, unlike $\beta$-strand- $\beta$-strand interactions, where association occurs via specific backbone hydrogen bonds, the helical interface does not provide chemically specific anchors for

\section{Significance}

Computational protein design tools use a bottom-up approach that allows for the testing of hypotheses on the relationships between amino acid sequence, protein structure and stability, and biological function. Here, we exploited two computational methods, protein docking and protein sequence optimization, to create a favorable protein-protein interaction between two identical proteins, resulting in a novel homodimer. A stepwise approach proved useful: scaffold stabilization followed by interface design to achieve homodimerization. Our results suggest that for some proteins, stabilization may be required for the successful design of functionality.

Author contributions: Y.M., P.-S.H., and S.L.M. designed research; Y.M., P.-S.H., F.-C.H., and S.-J.H. performed research; Y.M., P.-S.H., F.-C.H., S.-J.H., and S.L.M. analyzed data; and Y.M., P.-S.H., S.-J.H., and S.L.M. wrote the paper.

The authors declare no conflict of interest.

Freely available online through the PNAS open access option.

Data deposition: The atomic coordinates have been deposited in the Protein Data Bank, www.pdb.org (PDB ID codes 2MG4 and 4NDL)

${ }^{1}$ Y.M. and P.-S.H. contributed equally to this work

${ }^{2}$ Present address: Department of Biochemistry, University of Washington, Seattle WA 98195.

${ }^{3}$ To whom correspondence may be addressed. Email: shingjonghuang@ntu.edu.tw or steve@mayo.caltech.edu.

This article contains supporting information online at www.pnas.org/lookup/suppl/doi:10 1073/pnas.1505072112/-/DCSupplemental. 
protein-protein interactions. Although there are empirical rules for archetypal coiled-coil oligomerization (27), a general sequencestructure relationship that could be applied to an arbitrary scaffold has not yet been found. Furthermore, as shown by Keating and coworkers (28), predicting parallel or antiparallel helix-helix homodimers using computational modeling is challenging.

The similarity between parallel and antiparallel helix-helix structures and the high hydrophobicity of homodimers make it difficult to distinguish between the different conformational states, particularly if they are strongly competing with each other and only one of the states is explicitly designed. For example, Karanicolas et al. (29) computationally designed a novel protein-protein interface with tightly packed hydrophobic residues. The crystal structure, however, revealed that the orientation of one of the partners was rotated almost $180^{\circ}$ relative to its position in the design model. These results underscore the difficulty of excluding unwanted competing states in the design of protein-protein interactions.

Here, we design a C2-symmetric homodimer from a helical monomeric protein, Drosophila melanogaster engrailed homeodomain $(\mathrm{ENH})$. This small helix-turn-helix protein domain binds a specific sequence of dsDNA (30) and has been used as a model for theoretical and computational studies (31-33). Computational protein design (CPD) endeavors often begin with a thermostable scaffold because designs for targeted function (e.g., catalytic activity, ligand-protein binding affinity) have been shown to decrease protein stability significantly (34). Poor stability, in turn, often results in aggregation and can be problematic for protein expression and/or experimental characterization. The 51-aa fragment of WT ENH used in this study has a melting temperature $\left(\mathrm{T}_{\mathrm{m}}\right)$ of $49^{\circ} \mathrm{C}(35)$, which is low for a design scaffold. Indeed, we found that our initial attempt to build a homodimer using this scaffold resulted in a protein, ENH-c2a, that expresses in inclusion bodies even at temperatures as low as $16{ }^{\circ} \mathrm{C}$. For subsequent designs, we used the thermostabilized $\mathrm{ENH}$ variant NC3-NCap, a computationally designed protein that has a $\mathrm{T}_{\mathrm{m}}$ of $89^{\circ} \mathrm{C}(35)$. We applied a symmetric docking program based on a fast Fourier transform (FFT) algorithm (36) and designed the interface between four helices (two from each molecule) so that they would associate as a four-helix bundle. The final design (ENH-c2b) was experimentally characterized and shown to be a monodisperse homodimer with a $K_{\mathrm{d}}$ of $\sim 130 \mathrm{nM}$. The solution NMR structure reveals that the helical interface exhibits parallel packing consistent with the design model.

\section{Results}

Scaffold Selection. Due to our incomplete understanding of structure-function relationships and limitations in our ability to model proteins accurately, the success rate for CPD is generally rather low. This low success rate is particularly true for the de novo design of functional proteins, such as enzymes or proteins designed to interact with a specific target (37). Many de novo design studies therefore use a comprehensive approach that screens in silico scaffolds in the PDB, often resulting in tens to hundreds of candidate scaffolds $(12,16,38,39)$. These candidates are then used as the basis for computational design, with the top designs screened experimentally (e.g., for target binding affinity or catalytic activity). This approach has the advantage of providing a large amount of data that can be used to test design protocols $(22,40)$. Alternatively, focusing on a single scaffold allows the design to take specific features of the scaffold into account. For example, Privett et al. (34) used an iterative approach on a single scaffold that resulted in the de novo design of a Kemp eliminase that was further optimized by directed evolution to yield an enzyme with native-like kinetic parameters (41). ENH was selected as the target scaffold for this study because of its ability to bind to DNA, which could be exploited in future designs to create protein-DNA nanomaterials that self-assemble through noncovalent interactions. ENH binds DNA primarily with its third helix, leaving the surfaces of the first two helices available for design (30).

Design Protocol. Fig. 1 shows the steps we used to design and characterize a C2-symmetric homodimer. After selecting the scaffold protein, the surface side chains were pruned to $C \beta$ and the atomic radii were parameterized based on known $\mathrm{C} 2$ symmetry homodimers. We then applied a docking procedure, constrained for $\mathrm{C} 2$ symmetry, to generate initial models based on an FFT algorithm (36). FFT docking allowed for the efficient search of the translational and rotational $6 \mathrm{df}$. Approximately $10^{10}$ models were screened and ranked by shape complementarity. The top 200 candidates were clustered into 11 groups according to the pairwise rmsd values of the structures. Finally, these clusters were visually inspected, and one model was chosen for homodimer design. In the selected homodimer model, the first helix of each monomer pairs to form a parallel packing arrangement with the helices separated by $10 \AA$, similar to the 9.8- $\AA$ separation found in naturally occurring coiled-coil dimers (42) even though the design process did not explicitly consider the heptad repeat rules that govern coiled-coil interactions (24). Taken together, the first two helices of each monomer form a four-helix bundle structure (Fig. 1).

Symmetric sequence optimizations were applied to the 22 interfacial residues (11 on each side) of the homodimer model. To recapitulate the balance between nonpolar $(\sim 65 \%)$ and polar $(\sim 35 \%)$ amino acids seen in natural homodimers $(23)$, the value of the force-field parameter most responsible for limiting the burial of polar surface area, $\sigma_{p}$, was doubled relative to its standard setting (43).

Initial homodimer design was performed using WT ENH as the starting scaffold, resulting in a protein, ENH-c2a (Table 1), that could only be expressed in inclusion bodies (Fig. S1). Secondgeneration designs were performed using a previously designed thermostabilized variant of ENH, NC3-NCap, for the scaffold (35). A computationally designed library consisting of 128 variants of NC3-NCap was generated (Table S1), fused to YFP, and screened using a homo-FRET assay (Fig. S2A). Two variants resulting from the screen were characterized via size exclusion chromatography and analytical ultracentrifugation sedimentation velocity experiments; one proved to be a dimer, and the other was a tetramer (Fig. S2 $B$ and $C$ ). In a related paper (44), we

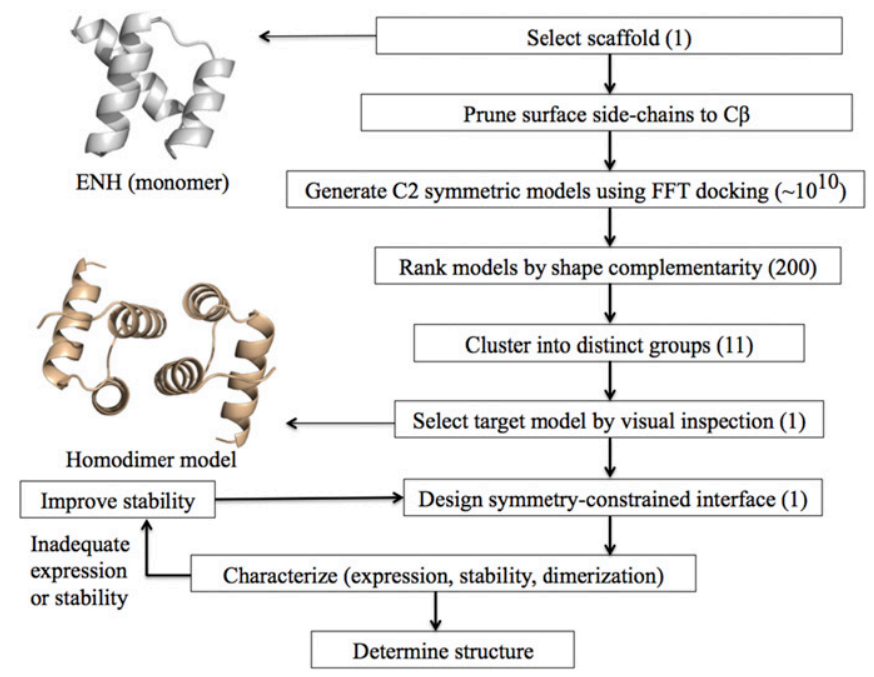

Fig. 1. Steps used to design the C2-symmetric homodimer. The initial scaffold, ENH, used for docking is shown in gray, and the homodimer model used for all interface designs is shown in bronze. The number of models created in each step is given in parentheses. 
Table 1. Sequences of WT ENH, ENH-c2a, NC3-Ncap, and ENH-c2b

НHHHHHHHHHHHH HHHHHHHHHH HHHHHHHHHHHHHHH

\begin{tabular}{|c|c|c|c|}
\hline Name & 123456789012345678901234567890123456789012345678901 & ID*, \% & $\mathrm{T}_{\mathrm{m}}$ \\
\hline $\mathrm{ENH}(\mathrm{WT})$ & TAFSSEQLARLKREFNENRYLTERRRQQLSSELGLNEAQIKIWFQNKRAKI & - & 49 \\
\hline $\mathrm{ENH}-\mathrm{C} 2 \mathrm{a}$ & $-\mathrm{KA}-\mathrm{DL}-\mathrm{YF}-\mathrm{W}-\mathrm{Y}-\mathrm{R}-$ & 82 & $\mathrm{n} / \mathrm{a}$ \\
\hline NC3-Ncap & -E-E-KR-DE-RRD-R-E-RD-QK-E-ER-RR-EQQ- & 55 & 88 \\
\hline $\mathrm{ENH}-\mathrm{C} 2 \mathrm{~b}$ & -E-E-KKA-DLA-YFD-R-PEW-RY-QR-E-ER-RR-EQQ- & 47 & 62 \\
\hline
\end{tabular}

The " $\mathrm{H}$ " series at the top shows the locations of the three helices in the ENH WT fold. n/a, not available. *ID is the sequence identity compared with WT ENH.

showed that the dimer, ENH_DsD, is domain-swapped and that it could be converted to a non-domain-swapped dimer through the introduction of proline at residue 23 . The resulting sequence, ENH$\mathrm{c} 2 \mathrm{~b}$ (Table 1), expressed well in the soluble fraction.

Biophysical Characterization of ENH-c2b. The designed proteins were characterized for soluble expression, secondary structure, thermostability, and oligomeric state. SDS/PAGE gels of purified $\mathrm{ENH}, \mathrm{ENH}-\mathrm{c} 2 \mathrm{a}$, and $\mathrm{ENH}-\mathrm{c} 2 \mathrm{~b}$ are shown in Fig. S1. Although ENH could be expressed in the soluble fraction at $16{ }^{\circ} \mathrm{C}$, ENH-c2a showed no soluble expression under the same conditions. In contrast, ENH-c2b expressed well at $37^{\circ} \mathrm{C}$, with yields of over $5 \mathrm{mg} / \mathrm{L}$ culture. CD spectroscopy revealed that $\mathrm{ENH}-\mathrm{c} 2 \mathrm{~b}$ is helical, folds reversibly, and has a $\mathrm{T}_{\mathrm{m}}$ of $\sim 62{ }^{\circ} \mathrm{C}$ (Fig. 2). Introduction of the designed interface reduced the $\mathrm{T}_{\mathrm{m}}$ of the scaffold by $\sim 26^{\circ} \mathrm{C}$ (Table 1 ).

Analytical ultracentrifugation sedimentation velocity experiments showed that ENH-c2b is a monodisperse dimer at a concentration of $5 \mu \mathrm{M}$ (Fig. $3 A$ ). The $K_{\mathrm{d}}$ for dimer formation was determined using a tryptophan fluorescence-based homo-FRET assay and was found to be $129 \pm 64 \mathrm{nM}$ (Fig. 3B), which is similar to the $K_{\mathrm{d}}$ values reported for other de novo-designed protein interfaces (before affinity maturation) $(12,13,16)$.

Structure Determination of ENH-c2b. X-ray crystallography attempts using an ENH_c2b variant with an extended and Histagged $\mathrm{N}$ terminus derived from the expression vector (pET28a) resulted in a dimer structure dominated by crystal packing not matching the design target and an interface area in the design region of only $699 \AA^{2}$ (Fig. S3 and Table S2). Heteronuclear single quantum coherence (HSQC) NMR of ${ }^{13} \mathrm{C}$ and ${ }^{15} \mathrm{~N}$ uniformly labeled, freshly prepared ENH-c2b showed a well-folded protein with sharp peaks that broadened over time. Adding six WT ENH amino acids (MEKRPR) at the $\mathrm{N}$ terminus, a Gly, and an eight-residue Strep-tag II at the C terminus of ENH-c2b (ENH-c2b-Strep) greatly enhanced its long-term stability. All NMR spectra showed only one set of chemical shifts for all residues, reflecting the underlying symmetry of ENH-c2b-Strep. Unambiguous chemical shift assignments were obtained for most of the backbone nuclei; however, peaks were missing for the last three residues, 21-23 (FYF), at the end of helix-1. This finding is consistent with the fact that the chemical shifts of aromatic residues are highly sensitive to their side-chain conformations and can be easily broadened if multiple conformations exchange on the NMR time scale (45).

We determined the structure of the ENH-c2b-Strep homodimer using $\psi / \varphi$ angle, hydrogen-bond, NOE, and C2-symmetry restraints (Table S3). Final coordinates of 10 models were deposited in the PDB with ID code 2MG4 (Fig. 4). The overall completeness of all (intramolecular and intermolecular) NOE restraints is $57 \%, 37 \%$, and $22 \%$ at $3-\AA, 4-\AA$, and $5-\AA$ cutoff distances, respectively (Table S4). For comparison, the average completeness for the 97 entries reported by Doreleijers et al. (46) is $68 \pm 14 \%, 48 \pm 13 \%$, and $26 \pm 9 \%$, at $3-\AA, 4-\AA$, and $5-\AA$ cutoff distances, respectively. There are 26 and 33 unambiguous intermolecular NOE restraints that have zero or one violation, respectively, in the 10 models of the NMR ensemble (Table S5). The intermolecular completeness of NOE restraints is $52 \%$ at a 4- $\AA$ cutoff distance (Table S6) compared with the average value of $37 \%$ reported by Doreleijers et al. (46).

Each monomeric subunit superimposes with WT ENH with an overall backbone rmsd of $2.5 \AA$, with the greatest deviation for the loop between helix-1 and helix-2, residues 21-23 (at the end of helix-1), and the $\mathrm{N}$ terminus (Fig. $5 A$ and $B$ ), which corresponds to regions lacking restraints and that show the greatest structural variation in the NMR ensemble (Fig. 4). The helices comprising the dimer interface, helix-1 and helix-2, align well with the design model (Fig. $5 A$ ), whereas the orientation of helix3 deviates from the orientation of the model (Fig. $5 B$ ). Compared with ENH, the most structurally defined regions of ENHc2b-Strep (helix-1, helix-2, helix-3, and the loop between helix-2 and helix-3) have a backbone rmsd of $1.4 \AA$.

The solution structure of the ENH-c2b-Strep dimer shows parallel helix-helix packing between helix-1 of each subunit, as in the homodimer design model. Compared with the model, the backbone rmsd of the most structurally defined regions (as discussed above) is $2.2 \AA$ (Fig. $5 C$ and $D$ ). The axial orientations of the four helices (helix- 1 and helix-2 from each subunit) are nearly identical with those helices in the model (Fig. $5 C$ ). The interface area is $2,189 \AA^{2}$, which falls in the range of natural single-patch homodimers $\left(2,740 \pm 1,240 \AA^{2}\right)(23)$. Nonpolar residues constitute $62 \%$ of the interface, close to the average value of $65 \%$ for natural homodimers (23). A NOESY experiment designed to retain only intermolecular interactions revealed several nonpolar interfacial residues that are likely to be important for dimerization, including Ala16, Leu19, Ala20, and Leu39 (Fig. 6). Structural alignment of only one of the subunits of the dimer emphasizes the differences between the structure of ENH-c2bStrep and the design model (Fig. $5 E$ and $F$ ).

\section{Discussion}

Stranges and Kuhlman (21) recently reviewed the computational design of novel protein-protein interfaces and pointed out the
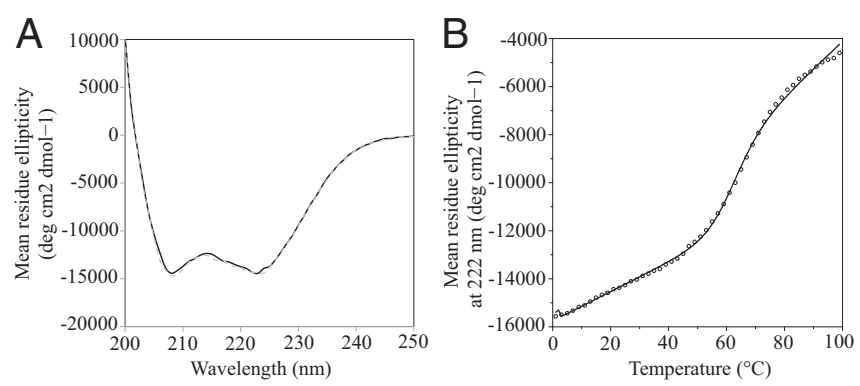

Fig. 2. $\mathrm{CD}$ analysis of ENH-c2b. $(A) \mathrm{CD}$ spectrum of ENH-c2b at room temperature: solid line, before thermal denaturation; dashed line, after thermal denaturation. $(B)$ Thermal denaturation curve measured at $222 \mathrm{~nm}$ : open circles, experimental data; line, fitted curve obtained using a two-state transition model. 

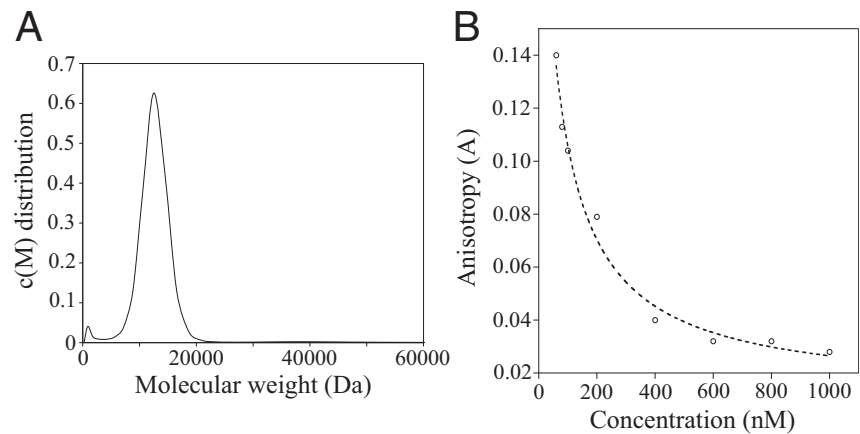

Fig. 3. Characterization of ENH-c2b oligomeric. (A) Sedimentation velocity experiment at $5 \mu \mathrm{M}$ with curve fit using the $c(\mathrm{M})$ (continuous distribution of molecular mass) model. (B) Tryptophan homo-FRET assay: circles, experimental data; dashed line, curve fit obtained using a monomer-dimer equilibrium model.

challenges this burgeoning field faces (21). Of 147 protein-protein interaction designs, only four were confirmed successful by X-ray crystallography (i.e., the solved structure matched the design model). All of the successful designs shared a number of common features: They exhibited fewer polar atoms $(<40 \%)$ and fewer buried hydrogen bonds at the designed interface than those polar atoms and buried hydrogen bonds seen in the failed designs. This reduced number of buried hydrogen bonds in the successful designs is in contrast to what is typically observed in natural dimers. Our homodimer design had similar characteristics: $62 \%$ nonpolar atoms and no buried $\mathrm{H}$-bonds at the interface. This higher interfacial hydrophobicity is expected, given that three of the five successful cases (including the one reported here) were designs for homodimers $(13,16)$, which naturally exhibit high nonpolar content at the interface (23). The other two successful cases (both heterodimer designs) also exhibited high interfacial hydrophobicity $(73 \%$ and $74 \%)$, which is likely related to the natural hydrophobicity of the targeted binding surface, the stem region of influenza HA $(12,15)$. However, designing largely hydrophobic binding surfaces can lead to failure in achieving the targeted structure. For example, the designed protein could bind to the target protein in the wrong orientation (29) or bind to itself to form undesired oligomers (12). In a case described in a related paper (44), when fused with YFP at the C terminus, ENH-c2b forms an unexpected but welldefined homodimer with dimerization interfaces formed between the ENH fragment and YFP. In addition, ENH-c2b with an extended $\mathrm{N}$-terminal tag forms a dimer with an interface that appears to be dominated by crystal packing forces (as described above). Nonetheless, successful designs of protein-protein interfaces based largely on hydrophobic interactions do exist, whereas the successful design of largely polar interfaces has yet to be fully demonstrated. Recently, Procko et al. (47) designed a protein inhibitor that binds to a hydrophilic patch on lysozyme with high affinity; however, this complex has yet to be structurally validated.

An interesting feature of the five successful designs is that the designed interfaces mainly involve the association of welldefined elements of secondary structures. Both of the successful HA heterodimer designs described above have "hot spots" on their helices that bind to helical structures on the target HA stem. Of the three successful homodimer designs to date, one is between two helices (this study), one involves metal-protein interactions mediated by helices (13), and one uses two exposed $\beta$-strands to form the homodimer (16). Loops can also be exploited in protein-protein interactions, as demonstrated by the widespread use of loops in antibody-antigen interactions. Many computational loop designs have been attempted; however, thus far, none have resulted in dimerization (12). In a communitywide assessment of protein-protein designs, Haliloglu and coworkers (22) found that many of the failed designs contain more loops and turns than successful designs, and that the higher flexibility of loops makes adopting a particular designed conformation difficult. Noninterfacial loop designs have demonstrated success (48-50). However, the design of interfaces involving loops appears to be more challenging, because the recognition-induced conformational changes that loops can undergo upon association with another protein are still poorly understood, and hence poorly modeled.

A significant difference between the design reported here and the four successful designs reported previously is that the accuracy of our design, with an rmsd of $2.2 \AA$, is lower than the 1.0 - to $1.80-\AA$ rmsd range reported for the other designs. The success of the four more accurate designs may be due to their incorporation of specific hot-spot residues or anchoring interactions that steered the formation of a high-affinity dimer. For example, the two HA heterodimer designs used predefined hot-spot residues to match the specified locations on the target patch (12) and the homodimer designs used either $\beta$-strand-mediated hydrogen bonds (16) or metal chelation to anchor the homodimerization (13). All of these designs exploited very specific pairwise interactions to facilitate and guide complex formation, which could be a general model that will be useful for future work.

As noted above, our initial design attempts using the WT $\mathrm{ENH}$ sequence as the parent for incorporating CPD-directed mutations resulted in a variant, ENH-c2a, that was not solubly expressed even at reduced temperatures, presumably because the introduced mutations compromised the stability of the protein. Introduction of a scaffold stabilization step into the design process (Fig. 1) led to the use of NC3-Ncap, a previously reported stabilized variant of ENH (35) that was able to support the mutations designed to confer homodimerization successfully. Thus, at least in this case, scaffold stabilization facilitated successful functional design. CPD has proved to be useful for designing proteins with improved stability (e.g., 51, 52). Designing for function, such as binding or catalytic activity, however, can lead to significant protein destabilization (34). In one example, Fleishman and coworkers (5) designed 88 proteins to bind to HA and found that $50 \%$ of them could not be solubly expressed in Escherichia coli. In the absence of highly stabilized scaffolds,

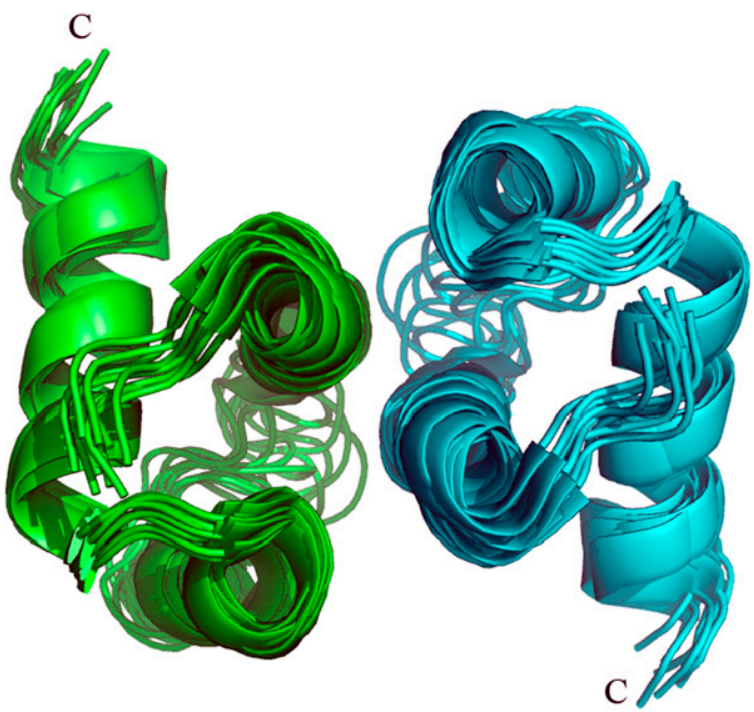

Fig. 4. Solution NMR structure ensemble showing the 10 lowest energy models for the core 51 amino acids (PDB ID code 2MG4). C, C terminus. 
A

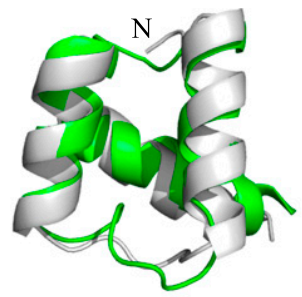

C

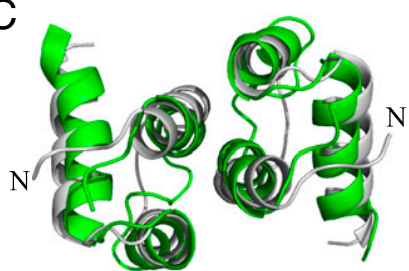

E

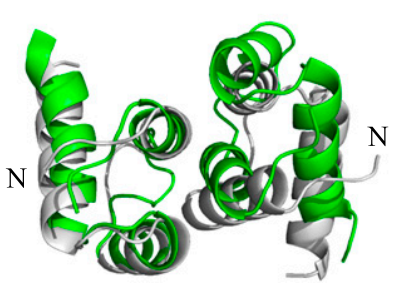

B

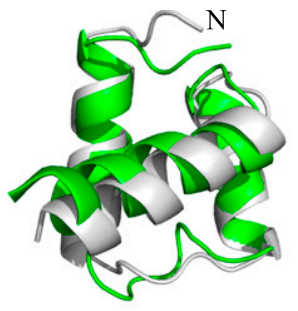

$\mathrm{D}$

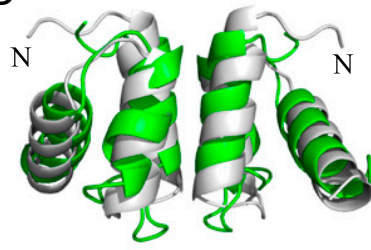

$\mathrm{F}$

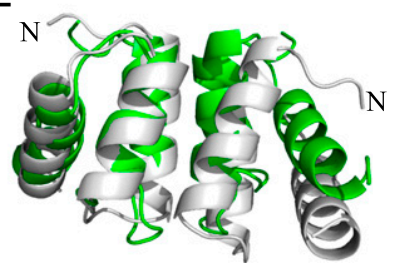

Fig. 5. Comparison of ENH-c2b averaged minimized solution NMR structure (green) and design model (gray). (A) Superposition of a single chain from the NMR structure and design model. (B) Alternative view of $A: \sim 180^{\circ}$ of rotation about the vertical axis. (C) Superposition of the entire dimer NMR structure and design model. $(D)$ Alternative view of $C: \sim 90^{\circ}$ of rotation about the horizontal axis. $(E)$ Superposition of the left chain of the NMR structure with the left chain of the design model showing the entire dimer structure. $(F)$ Alternative view of $E$ : $\sim 90^{\circ}$ of rotation about the horizontal axis. N, N terminus.

designs targeting the introduction or modification of protein function are likely to benefit from an initial round of CPDdirected scaffold stabilization.

\section{Conclusions}

This work represents an early example of the de novo design of a C2-symmetry homodimer via helical interactions. The successful design, ENH-c2b, is a monodisperse dimer with a $K_{\mathrm{d}}$ of $\sim 130 \mathrm{nM}$. The solution NMR structure is generally consistent with the design model with the protein-protein interface forming a fourhelix bundle. The homodimer design was achieved using a twostep computational approach in which the WT protein, ENH, was first stabilized and subsequently homodimerized. The final design, ENH-c2b, is $13{ }^{\circ} \mathrm{C}$ more stable than the WT ENH fragment used as the design scaffold and has a sequence identity of $47 \%$ compared with WT ENH.

\section{Materials and Methods}

Protein Docking and Computational Design. The ENH crystal structure (PDB ID code $1 \mathrm{ENH}$ ) was used as the scaffold for homodimerization, with side-chain atoms beyond $C_{\beta}$ deleted and atomic radii of the remaining atoms adjusted as follows: $\mathrm{N}, 1.4 \AA \AA^{\prime} \mathrm{O}, 1.3 \AA \mathrm{C}^{\prime}, 1.75 \AA \mathrm{C}_{\alpha}, 2.35 \AA$; and $\mathrm{C}_{\beta}$, $2.15 \AA$. A symmetric docking program based on an FFT algorithm was applied. One highscoring model was selected for computational designs. ORBIT CPD software was used for stability designs for both ENH and NC3-NCap. Initial interface designs were also done using ORBIT, and subsequent designs and analyses were done using our improved CPD programs PHOENIX and TRIAD. Details are provided in SI Methods.

Protein Expression and Purification. All proteins were expressed using BL21 DE3 cells transformed by pET plasmids with $1 \mathrm{mM}$ isopropyl $\beta$-D-1- thiogalactopyanoside in standard LB at $16{ }^{\circ} \mathrm{C}$ ( $\mathrm{His}_{6}-\mathrm{ENH}$ and ENH-C2a-Strep) or $37{ }^{\circ} \mathrm{C}$ (all other proteins). The ${ }^{13} \mathrm{C} /{ }^{15} \mathrm{~N}$-labeled ENH-c2b-Strep for NMR experiments was prepared by growing BL21 DE3 cells in $1 \mathrm{~L}$ of LB until the $\mathrm{OD}_{600}$ reached $\sim 0.6$ and transferring the cells to $250 \mathrm{~mL}$ of $\mathrm{M9}$ medium with ${ }^{13} \mathrm{C}$ glucose and ${ }^{15} \mathrm{~N}$ ammonium chloride. Purification of ENH-c2b was accomplished by fusing it to $\mathrm{His}_{6}$-ubiquitin, running the construct on an $\mathrm{Ni}^{2+}$-NTA column (Qiagen), and then cleaving $\mathrm{His}_{6}$-ubiquitin off using ubiquitin carboxyl-terminal hydrolase isozyme $\mathrm{L} 3$ protease $\left(37^{\circ} \mathrm{C}\right.$ overnight). Strep-Tactin Sepharose (IBA) and Superdex 75 (Amersham Pharmacia) columns were used for Strep-tag affinity chromatography and size exclusion chromatography, respectively. Details are provided in SI Methods.

CD Spectroscopy. CD studies were performed on an Aviv 62A DS spectropolarimeter equipped with a thermoelectric temperature controller Samples were prepared in $100 \mathrm{mM}$ sodium chloride and $20 \mathrm{mM}$ sodium phosphate buffer at $\mathrm{pH}$ 7.5. Wavelength scans and temperature denaturations were carried out in cuvettes with a $0.1-\mathrm{cm}$ pathlength at a protein concentration of $\sim 10 \mu \mathrm{M}$. Three wavelength scans were performed at $25^{\circ} \mathrm{C}$ for each sample and averaged. The thermal denaturation curve was collected at $222 \mathrm{~nm}$ from 0 to $99{ }^{\circ} \mathrm{C}$, sampling every $1{ }^{\circ} \mathrm{C}$ separated by 2 -min equilibration times (signal averaging time was $1 \mathrm{~s}$ ). The refolding curve was collected after the thermal denaturation experiment using the same sample.

Analytical Ultracentrifugation. ENH-c2b was analyzed on an XL-1 analytical ultracentrifuge equipped with an AnTi60 rotor (Beckman Coulter). Twochannel Epon-filled centerpieces were used for the sedimentation velocity experiment. Cells were torqued to 130 pounds per inch and run at 60,000 $\mathrm{rpm}$. Data were acquired at $230 \mathrm{~nm}$ and $20^{\circ} \mathrm{C}$ in continuous mode. Data were first fit to the $c(s)$ model (continuous distribution of sedimentation coefficient) and then converted to the $\mathrm{c}(\mathrm{M})$ model (continuous distribution of molecular mass). Time-invariant noises and baseline offsets were corrected before fitting. A maximum entropy regularization confidence level of 0.95 was used in all of the size distribution analyses.

Fluorescence Polarization Assay. Fluorescence polarization was measured at room temperature with a Fluorolog-3 spectrofluorometer (HORIBA). ENH-c2b was serially diluted in buffer containing $100 \mathrm{mM} \mathrm{NaCl}$ and $20 \mathrm{mM}$ Tris. $\mathrm{HCl}$ at $\mathrm{pH}$ 8.0. Fluorescence anisotropy was measured for each sample, and the G-factor was determined individually. Data were analyzed according to a simple monomer-dimer equilibrium model and fit with KaleidaGraph software (Synergy Software). Polarization values for the completely monomeric and dimeric states were fit to be $251 \mathrm{~mA}$ and $12 \mathrm{~mA}$, respectively.

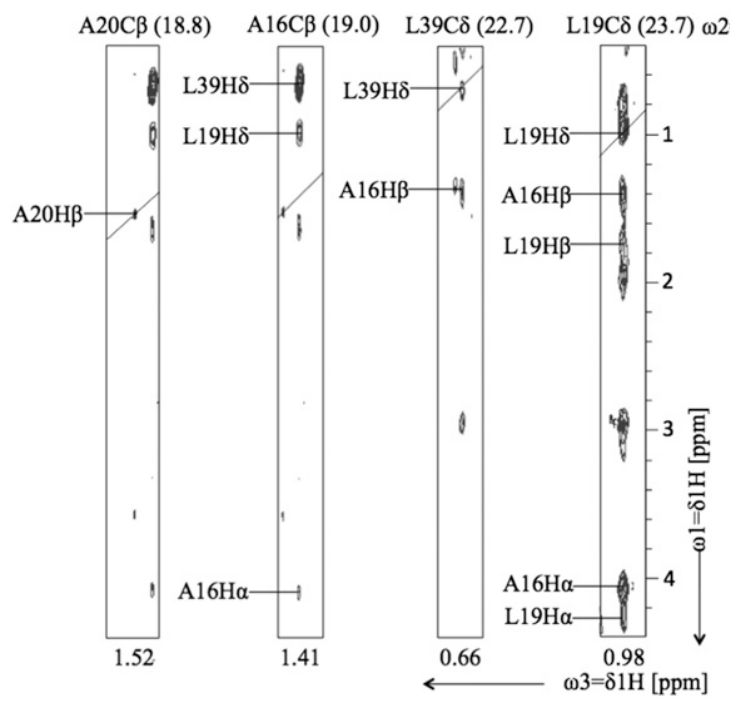

Fig. 6. NMR spectrum showing intermolecular NOE restraints obtained by a 3D ${ }^{13} \mathrm{C}^{15} \mathrm{~N}$-filtered NOESY- ${ }^{1} \mathrm{H}-{ }^{13} \mathrm{C}-\mathrm{HSQC}$ experiment. Contour plots of $\left[\omega_{1}\left({ }^{1} \mathrm{H}\right), \omega_{3}\left({ }^{1} \mathrm{H}\right)\right]$-strips of Ala20C ${ }_{\beta}$, Ala $16 C_{\beta}$, Leu39C $\mathrm{C}_{\delta}$ and Leu19 ${ }_{\delta}$ are shown. Chemical shifts indicated on the top and bottom correspond to $\omega_{2}\left({ }^{13} \mathrm{C}\right)$ and $\omega_{3}\left({ }^{1} \mathrm{H}\right)$ dimensions, respectively. For clarity, only the aliphatic region in the $\omega_{1}\left({ }^{1} \mathrm{H}\right)$ dimension is shown. Unambiguous restraints identified for Ala16, Ala20, Leu19, and Leu39 residues are labeled. 
Solution NMR Experiments. All spectra were acquired at $310 \mathrm{~K}$ on a Bruker Avance III 800 spectrometer equipped with a 5 -mm z-gradient TCI $\left({ }^{1} \mathrm{H},{ }^{13} \mathrm{C}\right.$, and ${ }^{15} \mathrm{~N}$ ) CryoProbe (Bruker). ENH-c2b-Strep $(1.9 \mathrm{mM}$ protein in $300 \mu \mathrm{L})$ was dissolved in $100 \mathrm{mM} \mathrm{NaCl}, 5 \mathrm{mM} \mathrm{CaCl}, 10 \mathrm{mM} \mathrm{DTT}, 0.02 \% \mathrm{NaN}_{3}, 5 \% \mathrm{D}_{2} \mathrm{O}$, and $20 \mathrm{mM} \mathrm{NH}_{4} \mathrm{OAc}$ at pH 4.5 in a Shigemi NMR tube. Assignment of mainchain and side-chain chemical shifts was based on ${ }^{1} \mathrm{H}-{ }^{15} \mathrm{~N}$ HSOC,${ }^{1} \mathrm{H}-{ }^{13} \mathrm{C}$ HSQC, CBCA(CO)NH, HNCACB, HNCO, HNCACO, HCCH-COSY, HCCH-TOCSY, HBHANH, HNHA(CO)NH, (H)CC(CO)NH, H(C)CCONH, HNHA, CACO, CON, and ${ }^{15} \mathrm{~N}$-TOCSY-HSQC experiments. NOE distance restraints were obtained from ${ }^{15} \mathrm{~N}$-edited NOESY, ${ }^{13} \mathrm{C}$-edited NOESY (aliphatic), and ${ }^{13} \mathrm{C}$-edited NOESY (aromatic) for intrachain or interchain contacts. An asymmetrically labeled dimer was prepared by mixing 1:1 uniformly ${ }^{13} \mathrm{Cl}^{15} \mathrm{~N}$-labeled and unlabeled

1. Hwang $H$, Vreven $T$, Janin J, Weng $Z$ (2010) Protein-protein docking benchmark version 4.0. Proteins 78(15):3111-3114.

2. Xu D, Tsai CJ, Nussinov R (1997) Hydrogen bonds and salt bridges across protein protein interfaces. Protein Eng 10(9):999-1012.

3. Bogan AA, Thorn KS (1998) Anatomy of hot spots in protein interfaces. $J$ Mol Biol 280(1):1-9.

4. Ritchie DW (2008) Recent progress and future directions in protein-protein docking Curr Protein Pept Sci 9(1):1-15.

5. Whitehead TA, Baker D, Fleishman SJ (2013) Computational design of novel protein binders and experimental affinity maturation. Methods Enzymol 523:1-19.

6. Halperin I, Ma B, Wolfson H, Nussinov R (2002) Principles of docking: An overview of search algorithms and a guide to scoring functions. Proteins 47(4):409-443.

7. Shifman JM, Mayo SL (2003) Exploring the origins of binding specificity through the computational redesign of calmodulin. Proc Natl Acad Sci USA 100(23):13274-13279.

8. Havranek JJ, Harbury PB (2003) Automated design of specificity in molecular recognition. Nat Struct Biol 10(1):45-52.

9. Sia SK, Kim PS (2003) Protein grafting of an HIV-1-inhibiting epitope. Proc Natl Acad Sci USA 100(17):9756-9761.

10. Lewis SM, Kuhlman BA (2011) Anchored design of protein-protein interfaces. PLoS One 6(6):e20872.

11. Procko E, et al. (2014) A computationally designed inhibitor of an Epstein-Barr viral Bcl-2 protein induces apoptosis in infected cells. Cell 157(7):1644-1656.

12. Fleishman SJ, et al. (2011) Computational design of proteins targeting the conserved stem region of influenza hemagglutinin. Science 332(6031):816-821.

13. Der BS, et al. (2012) Metal-mediated affinity and orientation specificity in a computationally designed protein homodimer. J Am Chem Soc 134(1):375-385

14. Sammond DW, et al. (2011) Computational design of the sequence and structure of a protein-binding peptide. J Am Chem Soc 133(12):4190-4192.

15. Whitehead TA, et al. (2012) Optimization of affinity, specificity and function of de signed influenza inhibitors using deep sequencing. Nat Biotechnol 30(6):543-548.

16. Stranges PB, Machius M, Miley MJ, Tripathy A, Kuhlman B (2011) Computationa design of a symmetric homodimer using $\beta$-strand assembly. Proc Natl Acad Sci USA 108(51):20562-20567.

17. King NP, et al. (2012) Computational design of self-assembling protein nanomaterials with atomic level accuracy. Science 336(6085):1171-1174

18. King NP, et al. (2014) Accurate design of co-assembling multi-component protein nanomaterials. Nature 510(7503):103-108.

19. Huang PS, et al. (2014) High thermodynamic stability of parametrically designed helical bundles. Science 346(6208):481-485.

20. Thomson AR, et al. (2014) Computational design of water-soluble $\alpha$-helical barrels Science 346(6208):485-488.

21. Stranges PB, Kuhlman B (2013) A comparison of successful and failed protein in terface designs highlights the challenges of designing buried hydrogen bonds. Protein Sci 22(1):74-82.

22. Fleishman SJ, et al. (2011) Community-wide assessment of protein-interface modeling suggests improvements to design methodology. J Mol Biol 414(2):289-302.

23. Bahadur RP, Chakrabarti P, Rodier F, Janin J (2003) Dissecting subunit interfaces in homodimeric proteins. Proteins 53(3):708-719.

24. Harbury PB, Zhang T, Kim PS, Alber T (1993) A switch between two-, three-, and fourstranded coiled coils in GCN4 leucine zipper mutants. Science 262(5138):1401-1407.

25. Grigoryan G, Reinke AW, Keating AE (2009) Design of protein-interaction specificity gives selective bZIP-binding peptides. Nature 458(7240):859-864

26. Guharoy M, Chakrabarti P (2007) Secondary structure based analysis and classification of biological interfaces: Identification of binding motifs in protein-protein in teractions. Bioinformatics 23(15):1909-1918.

27. Woolfson DN (2005) The design of coiled-coil structures and assemblies. Adv Protein Chem 70:79-112.

28. Apgar JR, Gutwin KN, Keating AE (2008) Predicting helix orientation for coiled-coil dimers. Proteins 72(3):1048-1065.
ENH-c2b. This sample was used for the $3 \mathrm{D}{ }^{13} \mathrm{C} /{ }^{15} \mathrm{~N}$-filtered NOESY- $-{ }^{1} \mathrm{H}-{ }^{13} \mathrm{C}-$ HSQC experiment (Fig. S4) to extract the interchain NOE restraints $(53,54)$. Details, including structure determination, are described in SI Methods.

ACKNOWLEDGMENTS. We thank Justin Chartron for useful discussion about solution NMR structural determination and Marie Ary for assistance with the manuscript. NMR measurements were carried out at Instrumentation Center of National Taiwan University, Taiwan (NSC 102-2731-M-002-002-MY2). This work was supported by the Defense Advanced Research Projects Agency Protein Design Processes Program, a National Security Science and Engineering Faculty Fellowship (NSSEFF N00244-09-1-0011, N00244-09-1-0082), and the Gordon and Betty Moore Foundation through Grant GBMF2809 to the Caltech Programmable Molecular Technology Initiative.

29. Karanicolas J, et al. (2011) A de novo protein binding pair by computational design and directed evolution. Mol Cell 42(2):250-260.

30. Fraenkel E, Rould MA, Chambers KA, Pabo CO (1998) Engrailed homeodomain-DNA complex at 2.2 A resolution: A detailed view of the interface and comparison with other engrailed structures. J Mol Biol 284(2):351-361.

31. Marshall SA, Mayo SL (2001) Achieving stability and conformational specificity in designed proteins via binary patterning. J Mol Biol 305(3):619-631.

32. Shah PS, et al. (2007) Full-sequence computational design and solution structure of a thermostable protein variant. J Mol Biol 372(1):1-6.

33. Beck DAC, Daggett $V$ (2004) Methods for molecular dynamics simulations of protein folding/unfolding in solution. Methods 34(1):112-120.

34. Privett HK, et al. (2012) Iterative approach to computational enzyme design. Proc Natl Acad Sci USA 109(10):3790-3795.

35. Marshall SA, Morgan CS, Mayo SL (2002) Electrostatics significantly affect the stability of designed homeodomain variants. J Mol Biol 316(1):189-199.

36. Huang P-S, Love JJ, Mayo SL (2005) Adaptation of a fast Fourier transform-based docking algorithm for protein design. J Comput Chem 26(12):1222-1232.

37. Khare SD, Fleishman SJ (2013) Emerging themes in the computational design of novel enzymes and protein-protein interfaces. FEBS Lett 587(8):1147-1154.

38. Röthlisberger $D$, et al. (2008) Kemp elimination catalysts by computational enzyme design. Nature 453(7192):190-195.

39. Jiang L, et al. (2008) De novo computational design of retro-aldol enzymes. Science 319(5868):1387-1391.

40. Kiss G, Röthlisberger D, Baker D, Houk KN (2010) Evaluation and ranking of enzyme designs. Protein Sci 19(9):1760-1773.

41. Blomberg R, et al. (2013) Precision is essential for efficient catalysis in an evolved Kemp eliminase. Nature 503(7476):418-421.

42. O'Shea EK, Klemm JD, Kim PS, Alber T (1991) X-ray structure of the GCN4 leucine zipper, a two-stranded, parallel coiled coil. Science 254(5031):539-544.

43. Dahiyat BI, Mayo SL (1996) Protein design automation. Protein Sci 5(5):895-903.

44. Mou Y, Huang P-S, Thomas LM, Mayo SL (2015) Using molecular dynamics simulations as an aid in the prediction of domain swapping of computationally designed protein variants. J Mol Biol, 10.1016/j.jmb.2015.06.006.

45. Wagner G, Brühwiler D, Wüthrich K (1987) Reinvestigation of the aromatic sidechains in the basic pancreatic trypsin inhibitor by heteronuclear two-dimensional nuclear magnetic resonance. J Mol Biol 196(1):227-231.

46. Doreleijers JF, Raves ML, Rullmann T, Kaptein R (1999) Completeness of NOEs in protein structure: A statistical analysis of NMR. J Biomol NMR 14(2):123-132.

47. Procko $E$, et al. (2013) Computational design of a protein-based enzyme inhibitor. $J$ Mol Biol 425(18):3563-3575.

48. Murphy PM, Bolduc JM, Gallaher JL, Stoddard BL, Baker D (2009) Alteration of enzyme specificity by computational loop remodeling and design. Proc Natl Acad Sci USA 106(23):9215-9220.

49. Mandell DJ, Coutsias EA, Kortemme T (2009) Sub-angstrom accuracy in protein loop reconstruction by robotics-inspired conformational sampling. Nat Methods 6(8): 551-552.

50. Hu X, Wang H, Ke H, Kuhlman B (2007) High-resolution design of a protein loop. Proc Natl Acad Sci USA 104(45):17668-17673.

51. Malakauskas SM, Mayo SL (1998) Design, structure and stability of a hyperthermophilic protein variant. Nat Struct Bio/ 5(6):470-475.

52. Korkegian A, Black ME, Baker D, Stoddard BL (2005) Computational thermostabilization of an enzyme. Science 308(5723):857-860.

53. Zwahlen C, et al. (1997) Methods for measurement of intermolecular NOEs by multinuclear NMR spectroscopy: Application to a bacteriophage lambda N-peptide/boxB RNA complex. J Am Chem Soc 119(29):6711-6721.

54. Breeze AL (2000) Isotope-filtered NMR methods for the study of biomolecular structure and interactions. Prog Nucl Magn Reson Spectrosc 36(4):323-372. 